\title{
Influencia de una aplicación de telefonía móvil de entrenamiento sobre las necesidades psicológicas y la motivación autodeterminada en escolares
}

\author{
Influence of a mobile telephone application for training about \\ psychological needs and autodetermined motivation in students
}

\section{Influência de um treinamento de aplicação de telefonia móvel em necessidades psicológicas e motivaçáo auto-determinada na escola}

\author{
Héctor Moreno-Casado ${ }^{1}$, Ricardo Cuevas ${ }^{2}$, Juan José Pulido González y Tomás García Calvo ${ }^{1}$
}

${ }^{1}$ Universidad de Extremadura (España) y ${ }^{2}$ Universidad de Castilla la Mancha (España).

Resumen: Sobre la base teórica de la Teoría de la Autodeterminación, con el presente estudio se pretendía analizar el efecto de las aplicaciones móviles relacionadas con el control del entrenamiento, sobre las necesidades psicológicas básicas, el nivel de autodeterminación hacia la práctica deportiva y la diversión. Para ello, se desarrolló un estudio cuasiexperimental en el que participaron 54 alumnos $(M=16.4 ; D T=1.51)$, de género masculino $(n=$ 32) y de género femenino $(n=22)$ de cinco centros de enseńanza secundaria de la comunidad autónoma de Extremadura. Los sujetos del grupo experimental $(n=27)$ desarrollaron un programa de intervención de entrenamiento autónomo de tipo aeróbico registrado y controlado en la aplicación móvil "Endomondo Sport Tracker" de ocho semanas de duración. Los componentes del grupo de control experimental $(n=27)$ registraron el mismo programa de entrenamiento autónomo en una ficha de registro facilitada por el grupo investigador. Los resultados demostraron que la satisfacción de la necesidad de competencia, la autodeterminación hacia la realización de la actividad y la diversión al realizarla fueron mayores en aquellos participantes que utilizaron la aplicación respecto a aquellos que no la utilizaron. A pesar de ello, no se registraron diferencias significativas en la mayoría de las variables analizadas en el estudio, por lo que se considera necesario seguir indagando en esta línea de trabajo.

Palabras clave: teoría de la autodeterminación, adolescentes, programa de intervención, actividad física.

Abstract: About the theoretical base of the Autodetermination Theory, the present research has tried to analyse the effect of the mobile applications which are related to the control of training about the basic psychological needs, the level of self-determination towards sport practice and fun. In order to do that, a quasi-experimental research was carried out in which 54 students $(M=16.4 ; S D=1.51)$, both male and female, of different state schools of secondary education of Extremadura. The subjects of the experimental group ( $n=27$ ) developed a programme of intervention of autonomous training of aerobic kind which was recorded and controlled with the mobile application "Endomondo Sport Tracker", with an eight- week duration. The components of the group of experimental control $(n=27)$ recorded the same programme of autonomous training in a record sheet provided by the research group. The results showed that the satisfaction of the need for competence, the self-determination towards the fulfillment of the activity and the fun when carrying it out were higher in those students who used the application in regard to those who did not. In spite of that, no meaningful differences were noticed in most of the variables analyzed in the study, therefore, deeper research in this field must be carried out.

Keywords: self-determination theory, adolescents, intervention program, physical activity.

Resumo: Com base teórica da Teoria da Autodeterminaçáo , o presente estudo teve como objetivo analisar o efeito de aplicaçōes móveis relacionados com a formação de monitoramento das necessidades psicológicas básicas , o nível de auto -determinação para o esporte e diversão., Sexo masculino ( $\mathrm{n}$ = 32) e feminino $(\mathrm{n}=22)$ de cinco escolas secundárias na comunidade; para fazer isso, um estudo quasi- experimental, em que 54 alunos ( $\mathrm{M}=1,51 \mathrm{DP}$ = 16,4 ) participaram desenvolvido Extremadura autônoma. Os indivíduos do grupo experimental $(\mathrm{n}=27$ ) desenvolveram um programa de intervenção treinamento aeróbio autônomo registrado e controlado nas "Endomondo Sports Tracker” aplicativo móvel oito semanas. Os componentes do grupo de controle experimental $(\mathrm{n}=27)$ tiveram o mesmo programa de treinamento autônomo em um formulário de inscrição fornecido pelo grupo de pesquisa Os resultados mostraram que a satisfação da necessidade de competência , auto -determinação para a realização da atividade e divertido de ser realizada foram maiores nos participantes que utilizaram o aplicativo com relação àqueles que não usaram . No entanto, não foram observadas diferenças significativas na maioria das variáveis analisadas no estudo, por isso é considerada necessária para continuar o trabalho nesta linha de trabalho.

Palavras-chave: teoria da autodeterminação, adolescentes, programa de intervenção, atividade física.
Uno de los principales objetivos de Educación Física en la etapa de Educación Secundaria es la instauración de hábitos

Dirección para correspondencia [Correspondence address]: Tomás García Calvo. Facultad de Ciencias del Deporte. Universidad de Extremadura. Avda. Universidad s/n. C.P. 10004 Cáceres (España). E-mail: tgarciac@unex.es de vida ligados a la actividad física fuera del ámbito escolar (Decreto 83/2007, de 24 de abril para Enseńanza Secundaria y Decreto 115/2008, de 7 de junio para Bachillerato). En la búsqueda de este objetivo, el profesorado suele percibir cierto rechazo hacia la realización de actividades físicas, más allá 
de las desarrolladas en la propia asignatura (Sánchez-Oliva, Leo, Sánchez-Miguel, Amado, y García-Calvo, 2013; Román, Serra-Majem, Macarro, Romero, y Torres, 2012). Este hecho se ha relacionado directamente con el abandono de la práctica (Cecchini et al. 2008). Conseguir dicho objetivo resulta fundamental, incluyendo la actividad física en la escala de valores del adolescente. Esta etapa evolutiva supone un periodo crítico en la adquisición de hábitos de vida para la edad adulta (Sánchez-Oliva, Amado, Chamorro, GonzálezPonce, y García-Calvo, 2013). Bajo esta premisa, diferentes estudios han destacado la importancia del tipo de motivación de alumnos y deportistas como elemento clave para conseguir una adecuada persistencia (Alvarez, Balaguer, Castillo, y Duda, 2009; García-Calvo, Sánchez-Miguel, Leo, SánchezOliva, y Amado, 2012; Moreno y González-Cutre, 2006).

En el estudio de las causas y circunstancias en las que se producen los comportamientos humanos, la marco-teoría de la Autodeterminación (Deci y Ryan, 2000; Ryan y Deci, 2000) es cada vez más utilizada en el análisis de los procesos motivacionales dentro del contexto de la actividad física y el deporte. Dentro de esta teoría, la micro-teoría de la integración orgánica (Deci y Ryan, 1995) señala que la motivación se puede explicar en torno a un continuo motivacional. En este continuo se pueden distinguir los diferentes niveles de autodeterminación: la motivación intrínseca, que representa el nivel más alto de autodeterminación. Se refiere al compromiso con una actividad por propia iniciativa, por el placer y por la satisfacción que supone la participación; la motivación extrínseca, que surge cuando una persona se involucra en una actividad para logar ciertos objetivos extrínsecos. No tiene un fin en sí mismo e involucra diferentes tipos de regulación, que de mayor a menor autodeterminación son: la regulación integrada, identificada, introyectada y externa. Por último, la desmotivación, que constituye el nivel más bajo de autodeterminación. Se caracteriza porque el sujeto no tiene intención de realizar una actividad. Por lo tanto, existe una falta total de motivación, tanto intrínseca como extrínseca.

Paralelamente, a través de la micro-teoría de las Necesidades Psicológicas Básicas (Ryan y Deci, 2000) estos autores indican que para que las personas tengan una motivación autodeterminada resulta indispensable tener cubiertas tres Necesidades Psicológicas Básicas. Estos tres mediadores fundamentales y universales entre los factores sociales (situacionales, contextuales y globales) y los niveles de motivación (Vallerand y Rousseau, 2001) son: la necesidad de autonomía (deseo de ser el origen de sus comportamientos), la necesidad de competencia (deseo de actuar eficazmente en el contexto que le rodea) y la necesidad de relaciones sociales (sensación de conexión con los individuos del entorno social).

Dentro de los elementos que generan una motivación autodeterminada en adolescentes, tradicionalmente se ha considerado que las nuevas tecnologías han desplazado de forma nociva a la actividad física en el uso de su tiempo libre (Janz y Mahoney, 1997; Motl, McAuley, Birnbaum, y Lytle, 2006). En cambio, otros estudios demuestran que pueden coexistir (Biddle, Gorely, Marshall, Murdey, y Cameron, 2003) o que incluso afectan de forma positiva a la realización de actividad física (Beltrán, Molina, y Valencia, 2011).

Explorando esta relación positiva, se han producido hasta ahora algunas intervenciones en el ámbito educativo. A nivel internacional, en Canadá se llevó a cabo el programa Daily Physical Activity in Elementary Schools (Naylor, Macdonald, Zebedee, Reed, y McKay, 2006). Este programa tenía como objetivo incluir e inculcar en la educación primaria la práctica de 20 minutos de actividad física diaria. En este caso, el uso de elementos tecnológicos (uso de emails, páginas webs para dar feedback a los alumnos, profesores y padres, empleo del vídeo para motivar a los alumnos...) junto a un trabajo conjunto de padres, profesores y alumnos en el fomento de la actividad física, permitió aumentar considerablemente la práctica en los jóvenes en edad escolar, además de la motivación hacia la práctica. Sobresale en España el estudio de Sigue la Huella (Murillo, García Bengoechea, Julián, y Generelo, 2014), mediante el uso de acelerómetros, el empleo del vídeo como medio para fomentar la práctica de la actividad física y el deporte y el análisis de los parámetros biológicos relacionados con la salud.

Dentro de las nuevas tecnologías, el desarrollo de smartphones y el uso de aplicaciones para telefonía móvil de control, análisis y publicación de la actividad física realizada por el sujeto se ha generalizado (Kranz et al., 2012). Este tipo de aplicaciones registran los entrenamientos (tiempo, distancia, velocidad, ritmo, calorías, altitud...). Además, trazan el mapa de los recorridos y establecen objetivos por tiempo y distancia, entre otros. Otra característica a destacar de este tipo de aplicación es la posibilidad de compartir los entrenamientos en las redes sociales (Facebook, Twitter). Concretamente, algunos estudios (Mulas, Pilloni, y Carta, 2012; Vickey y Breslin, 2011) han demostrado mayores relaciones positivas entre nuevas tecnologías y motivación y adherencia hacia la realización de actividad física.

No obstante, las investigaciones encontradas con intervenciones que aplicaron este tipo de tecnología son escasas. En ellas se han encontrado beneficios en la influencia de este tipo de tecnología sobre elementos relacionados con el estado de salud (Fanning, Mullen, y McAuley, 2012).

Por lo tanto, y a pesar de los resultados de estos trabajos, la reciente aparición de este tipo de recursos hace necesaria la puesta en marcha de nuevos estudios que traten de constatar el impacto que estas tecnologías pueden tener en los procesos motivacionales relacionados con la actividad físico-deportiva (King et al., 2013).

Con esta intención, en el presente trabajo se llevó a cabo una intervención con adolescentes, con grupo experimental y 
grupo de control, para tratar de determinar la influencia de una aplicación específica de entrenamiento sobre las necesidades psicológicas básicas y su nivel de autodeterminación. En este sentido, se planteó la siguiente hipótesis: el programa de actividad física basado en el uso de la aplicación móvil mejorará significativamente la satisfacción de las tres necesidades psicológicas básicas, la motivación autodeterminada y la diversión del alumnado del grupo experimental. Por otro lado, el programa de actividad física tradicional, que no utiliza aplicación móvil, mantendrá los mismos niveles de satisfacción de las necesidades psicológicas básicas, la motivación autodeterminada y la diversión y aburrimiento en los alumnos del grupo de control antes y después de la intervención.

\section{Método}

\section{Participantes}

Se utilizó un diseño cuasi-experimental con medidas pre- y post-test en el grupo experimental y en el de control, a los cuales les fueron asignados tratamientos diferentes. Formaron parte un total de 54 alumnos $(M=16.4 ; D T=1.51)$ de $4^{\circ}$ de $\mathrm{ESO}$, de $1^{\circ}$ y $2^{\circ}$ de Bachillerato de género masculino $(n=$ 32) y femenino $(n=22)$, de cinco centros educativos de enseñanza secundaria de la comunidad autónoma de Extremadura. La selección de la muestra se realizó intencionadamente por proximidad, así como por la existencia y disponibilidad de teléfonos móviles con la tecnología requerida. La asignación de los alumnos al grupo experimental y al de control se realizó de forma aleatoria.

\section{Procedimiento}

Para su participación, a todos los sujetos se les facilitó un consentimiento informado que cumplimentaron sus padres o tutores legales, autorizando su participación en esta investigación. Se informó del trato confidencial al ámbito de la propia investigación de todas sus respuestas y resultados. En todo momento fueron respetadas las normas éticas de actuación necesarias al trabajar con menores, así como los acuerdos de la Declaración de Helsinki (1964). El alumnado participante se encontraba con un nivel de aprendizaje adecuado de conocimiento y aplicación de los métodos de entrenamiento de las capacidades físicas básicas (Decreto 83/2007, de 24 de abril para Enseñanza Secundaria y Decreto 115/2008, de 7 de junio para Bachillerato). Con ello, se estaba en condiciones de poder desarrollar de forma autónoma las actividades que conformaban el programa de intervención de esta investigación.

Se establecieron dos grupos de trabajo: un grupo experimental, que utilizó la aplicación Endomondo Sport Tracker para registrar, controlar y publicar sus entrenamientos, formando parte del grupo de las GPS Tracker (Kranz et al.,
2012). La selección de esta aplicación móvil se debió fundamentalmente a sus amplias utilidades y a su carácter gratuito. Se trata de una aplicación que tiene una presentación y formato de uso muy intuitivo y en castellano, lo que le hace ser una opción muy cómoda para su uso en el rango de edad de la muestra.

Con el grupo experimental, se realizaron pruebas en el uso de la aplicación en teléfonos móviles de este tipo durante las dos semanas previas al inicio del programa de intervención, con el fin de familiarizar a los sujetos en su presentación y uso básico, así como para detectar e intentar resolver posibles incidencias, dudas o problemas en el uso de la aplicación.

Gestionado y controlado por el investigador principal de la investigación, se creó en redes sociales un perfil de Facebook en el que se procedió a incluir a todos los miembros del grupo experimental. En este perfil se publicaron los entrenamientos de cada sujeto del grupo experimental y no pudieron formar parte de él los miembros del grupo de control. El grupo de control reflejó sus entrenamientos en un modelo de ficha de registro facilitada por el grupo investigador antes del comienzo del programa de intervención. En ella, los componentes del grupo de control registraban tiempo, distancia, ritmo de entrenamiento, tipo de recorrido realizado, etc. Este registro en forma de ficha fue entregado al finalizar el programa de intervención de cada sujeto.

Del mismo modo, se aclaró de forma a los sujetos de uno y otro grupo que no podían compartir la realización de los entrenamientos durante el tiempo que durara el programa con sujetos del otro grupo.

Para llevar a cabo la recogida de datos de los participantes, se obtuvo la colaboración desinteresada del profesorado de educación física de cada uno de los centros participantes (mediciones, recogida de fichas de control de entrenamientos, comunicación de problemas o dudas surgidas, etc.). Previo a la recogida de datos, se facilitó a los profesores colaboradores de cada centro la información relativa a cómo recoger los datos. De este modo la obtención de datos fue homogénea en los diferentes centros, en constante comunicación con el grupo investigador. Todos los sujetos completaron de forma telemática los cuestionarios fuera del horario de clases, a través de un cuestionario creado para tal ocasión en Google Docs ${ }^{1}$, cuyo enlace web se facilitó a los correos electrónicos disponibles de cada uno de los alumnos.

A la hora de proceder a la medición de las variables, se realizaron dos medidas: una medida pre-, anterior al comienzo de la realización del programa de mejora de la resistencia aeróbica; y una medida post-, realizada al final del mismo, ocho semanas después. Dicho programa de intervención se carac-

1 Software libre que permite crear cuestionarios on-line, pudiendo acceder al documento a través de un enlace URL. Una vez completado el cuestionario, el software almacena los datos en un documento Excel. 
terizó por los siguientes aspectos: 1- Tipo de actividad física: La actividad física a desarrollar fue de tipo aeróbico, conocida y practicada por el alumnado participante (trote, paseo, carrera continua, cross-paseo...). 2- Duración total del programa: 8 semanas, estableciendo dos semanas previas de "rodaje" y prueba informal de la aplicación seleccionada, así como de conocimiento del modo de proceder en el registro del grupo de control. 3- Número de sesiones semanales: Se estableció como mínimo la realización de 3 sesiones semanales hasta un máximo de 4 sesiones, excluyendo de este número aquellas actividades ya realizadas por los sujetos con anterioridad a su participación en esta investigación (entrenamientos, competiciones deportivas, etc). 4-Características de las sesiones: Las sesiones debieron tener, con independencia de la distanciaritmo realizado, un mínimo de 20 minutos de duración y un máximo de 50 minutos, adaptando la exigencia de cada sesión de forma libre por parte del sujeto. 5- Objetivo individual: De forma individual, los objetivos a conseguir por cada sujeto se estimaron de forma porcentual sobre su nivel inicial, concretamente sobre los dos parámetros siguientes: $1^{\circ}$ - Tiempo: 20\% de aumento sobre el tiempo inicial o llegar al límite de 50 minutos establecido como máximo para cada sesión de entrenamiento; $2^{\circ}$ - Distancia recorrida: aumento del 30\% sobre la distancia inicial. Estos porcentajes se establecieron en función de las sesiones de la primera semana en comparación con las sesiones de la última semana. 6- Objetivo colectivo: Con el fin de crear un ambiente de relaciones interpersonales a partir de la actividad, se marcaron para cada uno de los centros participantes dos objetivos comunes para el grupo: 10-Tiempo: 35 minutos; $2^{o}$ - Distancia recorrida: Superar los 5 kilómetros. Estos valores se extrajeron de los valores medios de los alumnos que participaron en la investigación.

\section{Instrumentos}

Satisfacción de las Necesidades Psicológicas Básicas en el Ejercicio. Para valorar la satisfacción de las necesidades psicológicas básicas se utilizó la adaptación al castellano (Sánchez y Núñez, 2007) del cuestionario The Basic Psychological Needs in Exercise Scale-BPNES- (Vlachopoulos y Michailidou, 2006). Como frase introductoria esta escala aplica "Cuando hago actividades físicas aeróbicas (correr, montar en bici...)...”, a la que le siguieron 12 ítems, agrupados en tres factores (4 ítems cada uno): autonomía (ejemplo: "Creo fuertemente que tengo la oportunidad de tomar decisiones respecto a la mane- ra de hacerlas"), competencia (ejemplo : "Creo que progreso enormemente con respecto al objetivo final"), y relaciones sociales (ejemplo: "Me siento muy cómodo/a con los otros participantes").

Regulación motivacional. Para valorar la motivación, se empleó la versión en castellano (González-Cutre, Sicilia, y Fernández, 2010) del Behavioral Regulation in Exercise Questionnaire-3 (BREQ-3: Wilson, Rodgers, Loitz, y Scime, 2006). Esta escala se inicia con la frase introductoria "Yo hago ejercicio físico de tipo aeróbico...”, seguida de 23 ítems que miden los seis tipos de regulación motivacional: intrínseca (4 ítems, ejemplo: "Porque creo que el ejercicio aeróbico es divertido"), integrada (4 ítems, ejemplo: "Porque considero que el ejercicio físico aeróbico forma parte de mí"), identificada (3 ítems, ejemplo: "Porque valoro los beneficios que tiene el ejercicio físico aeróbico"), introyectada (4 ítems, ejemplo: "Porque me siento culpable cuando no lo practico"), externa (4 ítems, ejemplo: "Porque mis amigos/familia me dicen que debo hacerlo"), y desmotivación (4 ítems, ejemplo: "No veo el sentido de hacer ejercicio físico aeróbico").

Diversión. Para determinar el nivel de diversión, se aplicó la versión en castellano (Balaguer, Atienza, Castillo, Moreno, y Duda, 1997) del Cuestionario de Satisfacción Intrinseca en el Deporte (SSI: Duda y Nicholls, 1992), utilizando la versión española. La escala comienza por la frase: "Identifica tus sensaciones y estados de ánimo sobre las siguientes cuestiones:...", seguida de los 8 ítems de la escala, diferenciando los factores de diversión ( 5 ítems, ejemplo: "Cuando hago actividad aeróbica parece que el tiempo vuela") y aburrimiento (3 ítems, ejemplo: "Cuando practico actividades físicas aeróbicas normalmente me aburro").

El formato de respuesta empleado para todos los instrumentos fue tipo Likert, de 1 (totalmente en desacuerdo) a 5 (totalmente de acuerdo).

\section{Análisis de datos}

Para el tratamiento y análisis de los resultados se ha utilizado el programa estadístico SPSS 19.0, software registrado de IBM. En primer lugar, se llevó a cabo un análisis factorial exploratorio, un análisis confirmatorio y un análisis de fiabilidad para verificar la validez y consistencia interna de los diferentes factores del estudio, en el que se obtuvieron aceptables valores cercanos o superiores a .70 (Nunnally y Bernstein, 1994) cuyos valores se describen en la siguiente tabla: 
Tabla 1. Análisis de consistencia interna. Valores del alfa de Cronbach.

\begin{tabular}{lcccc}
\hline \multirow{2}{*}{ Variables } & \multicolumn{3}{c}{ Grupo Control } & \multicolumn{2}{c}{$\begin{array}{c}\text { Grupo } \\
\text { experimental }\end{array}$} \\
\cline { 2 - 5 } & Pre & Post & Pre & Post \\
\cline { 2 - 5 } & $A$ & $\alpha$ & $A$ & $A$ \\
\hline Satisfacción Autonomía &, 69 &, 58 &, 74 &, 76 \\
Satisfacción Competencia &, 78 &, 68 &, 71 &, 83 \\
Satisfacción Relaciones Sociales &, 66 &, 76 &, 73 &, 75 \\
Regulación Intrínseca &, 79 &, 68 &, 82 &, 84 \\
Regulación Integrada &, 79 &, 65 &, 65 &, 92 \\
Regulación Identificada &, 79 &, 44 &, 65 &, 74 \\
Regulación Introyectada &, 87 &, 76 &, 72 &, 74 \\
Regulación Externa &, 82 &, 69 &, 69 &, 66 \\
Desmotivación &, 87 &, 83 &, 57 &, 72 \\
Diversión &, 90 &, 47 &, 71 &, 82 \\
Aburrimiento &, 66 &, 72 &, 64 &, 84
\end{tabular}

Seguidamente, se realizó un análisis descriptivo y se llevó a cabo un ANOVA de medidas repetidas de un modelo de dos factores para tratar de conocer la influencia del programa de intervención sobre cada una de las variables en ambos grupos. Por último, para determinar las diferencias de cada grupo en función de la medida, se procedió a realizar un análisis de pruebas no paramétricas de los rangos con signo de Wilcoxon.

\section{Resultados}

\section{Análisis descriptivo}

En primer lugar, en la tabla 2 se muestran los estadísticos descriptivos de las diferentes variables incluidas en el estudio. Estos resultados mostraron medias muy elevadas en la satisfacción de las necesidades psicológicas básicas en ambos grupos. Del mismo modo, se registraron mayores valores en las regulaciones motivacionales más cercanas a los máximos niveles de autodeterminación respecto a las regulaciones más próximas a la desmotivación.

Tabla 2. Estadísticos descriptivos.

\begin{tabular}{|c|c|c|c|c|c|c|c|c|c|}
\hline \multirow{3}{*}{ Variables } & \multicolumn{4}{|c|}{ Grupo Control } & \multicolumn{4}{|c|}{ Grupo experimental } & \multirow[b]{3}{*}{ Range } \\
\hline & \multicolumn{2}{|c|}{ Pre } & \multicolumn{2}{|c|}{ Post } & \multicolumn{2}{|c|}{ Pre } & \multicolumn{2}{|c|}{ Post } & \\
\hline & $M$ & $D T$ & $M$ & DT & $M$ & $D T$ & M & DT & \\
\hline Satisfacción Autonomía & 3,98 &, 49 & 3,92 &, 65 & 3,93 &, 55 & 3,88 & ,64 & $1-5$ \\
\hline Satisfacción Competencia & 4,12 &, 52 & 4,10 & ,66 & 3,83 & ,63 & 3,91 &, 67 & $1-5$ \\
\hline Satisfacción Relaciones Sociales & 4,26 &, 68 & 4,21 &, 75 & 4,41 & ,67 & 4,31 &, 55 & $1-5$ \\
\hline Regulación Intrínseca & 4,13 & ,69 & 4,08 &, 67 & 4,01 & ,88 & 4,12 &, 72 & $1-5$ \\
\hline Regulación Integrada & 3,59 &, 85 & 3,84 &, 73 & 3,68 & 1,05 & 3,78 &, 78 & $1-5$ \\
\hline Regulación Identificada & 4,26 &, 65 & 4,34 &, 55 & 4,14 &, 40 & 4,32 &, 40 & $1-5$ \\
\hline Regulación Introyectada & 2,01 &, 72 & 2,27 & ,84 & 1,87 & ,94 & 2,20 &, 88 & $1-5$ \\
\hline Regulación Externa & 1,36 &, 58 & 1,30 & ,46 & 1,21 & 40 & 1,31 &, 40 & $1-5$ \\
\hline Desmotivación & 1,29 &, 39 & 1,37 & ,60 & 1,37 & 64 & 1,47 &, 72 & $1-5$ \\
\hline Diversión & 4,53 &, 55 & 4,56 &, 55 & 4,38 &, 77 & 4,48 &, 57 & $1-5$ \\
\hline Aburrimiento & 1,25 &, 52 & 1,53 & ,49 & 1,18 & ,39 & 1,62 &, 77 & $1-5$ \\
\hline
\end{tabular}

A la hora de observar la evolución de cada variable a lo largo del tiempo, se detectó que la satisfacción de la necesidad de competencia aumentó en el grupo experimental, mientras que disminuyó ligeramente en el grupo de control. Esta evolución no se produce en la satisfacción de las otras dos necesidades psicológicas básicas, donde se produce un descenso en los valores, siendo más acusado en la satisfacción de la necesidad de autonomía que en la de relaciones sociales. Por lo que se refiere las regulaciones motivacionales, el grupo experimental registró un aumento de todas ellas. En el grupo control, esta evolución positiva se produce también, a excepción de la regulación intrínseca y regulación externa. En la figura 1, se observa de forma gráfica la evolución de medias de la satisfacción de las necesidades psicológicas básicas.

\section{ANOVA de medidas repetidas}

Para verificar los efectos provocados por la interacción del tratamiento y el tiempo en cada una de las variables dependientes, se realizó un ANOVA de medidas repetidas. En la tabla 3 se muestran los resultados obtenidos, en los que se observan tendencias de mejora en algunas de las variables medidas pero sin cambios significativos. 
Tabla 3. ANOVA de medidas repetidas comparando grupo de control y experimental antes y después de la intervención.

\begin{tabular}{lllll}
\hline Variables & $M C$ & $F$ & $p$ & $\eta^{2}$ \\
\hline Satisfacción Autonomía &, 00 &, 00 &, 96 &, 00 \\
Satisfacción Competencia &, 08 &, 33 &, 57 &, 01 \\
Satisfacción Relaciones Sociales &, 00 &, 00 &, 97 &, 00 \\
Regulación Intrínseca &, 17 &, 53 &, 47 &, 01 \\
Regulación Integrada &, 15 &, 44 &, 51 &, 01 \\
Regulación Identificada &, 06 &, 23 &, 64 &, 00 \\
Regulación Introyectada &, 03 &, 11 &, 75 &, 00 \\
Regulación Externa &, 20 & 1,93 &, 17 &, 04 \\
Desmotivación &, 01 &, 02 &, 90 &, 00 \\
Diversión &, 03 &, 15 &, 70 &, 00 \\
Aburrimiento &, 19 &, 74 &, 39 &, 01 \\
\hline
\end{tabular}

${ }^{* *} p<.01 ;{ }^{*} p<.05$.

\section{Análisis de contraste}

Por último, se procedió a realizar un análisis de contraste utilizando para ello una prueba no paramétrica Wilcoxon debido al tamaño de la muestra. Los resultados encontrados no mostraron diferencias significativas entre la medida inicial y final en las satisfacciones de las necesidades psicológicas básicas en ambos grupos analizados, control y experimental. Seguidamente, considerando a las regulaciones motivacionales, tampoco se obtuvieron diferencias significativas en las medidas pre y post entre ambos grupos. Por último, teniendo en cuenta las variables de diversión y aburrimiento, se apreciaron diferencias significativas en el aburrimiento entre la medida pre y post, tanto en el grupo de control $(p<.05)$ como en el grupo experimental $(p<.05)$. Concretamente, se produjo un aumento del aburrimiento en la medida post tanto en el grupo de control, como en el grupo experimental.

Tabla 4. Análisis de estadísticos de contraste con prueba no paramétrica Wilcoxon.

\begin{tabular}{lcccc}
\hline \multirow{2}{*}{ Variables } & \multicolumn{2}{c}{$\begin{array}{c}\text { Grupo Control } \\
\text { Pre-Post- }\end{array}$} & \multicolumn{2}{c}{$\begin{array}{c}\text { Grupo Experimental } \\
\text { Pre-Post- }\end{array}$} \\
\cline { 2 - 5 } & $Z$ & $p$ & $Z$ & $P$ \\
\hline Autonomía &,- 15 &, 88 &,- 21 &, 83 \\
Competencia &,- 33 &, 73 &,- 63 &, 52 \\
Relaciones Sociales &,- 34 &, 73 &,- 58 &, 56 \\
Intrínseca &,- 22 &, 82 &,- 27 &, 78 \\
Integrada & 1,54 &, 12 &,- 76 &, 44 \\
Identificada &,- 83 &, 40 &,- 94 &, 34 \\
Introyectada & $-1,95$ &, $05^{*}$ & $-1,87$ &, 06 \\
Externa &, 63 &, 52 & $-1,35$ &, 17
\end{tabular}

\begin{tabular}{lcccc}
\hline \multirow{2}{*}{ Variables } & \multicolumn{2}{c}{$\begin{array}{c}\text { Grupo Control } \\
\text { Pre-Post- }\end{array}$} & \multicolumn{2}{c}{$\begin{array}{c}\text { Grupo Experimental } \\
\text { Pre-Post- }\end{array}$} \\
\cline { 2 - 5 } & $Z$ & $p$ & $Z$ & $P$ \\
\hline Desmotivación &,- 12 &, 76 &,- 57 &, 56 \\
Diversión &,- 18 &, 85 &,- 54 &, 58 \\
Aburrimiento & $-2,39$ &, $01^{* *}$ & $-2,49$ &, $01^{* *}$ \\
\hline${ }^{* *} p<.01 ;{ }^{*} p<.05$. & & & &
\end{tabular}

\section{Discusión}

El objetivo de este estudio era determinar la influencia que podían ejercer las nuevas tecnologías sobre las necesidades psicológicas básicas, la motivación y la diversión de escolares adolescentes a la realización de actividades físicas aeróbicas. La hipótesis del estudio enunciaba, en primer lugar, que la utilización de la aplicación "Endomondo Sport Tracker" sobre las necesidades psicológicas básicas en jóvenes escolares tendría un efecto positivo y mayor que su no utilización. Se pudo comprobar que la satisfacción de la necesidad de competencia aumentó, aunque no sucedió lo mismo en las otras dos necesidades psicológicas básicas. Estos resultados contradicen los hallazgos obtenidos por Kranz et al. (2012), que encontraron mejoras sustanciales en la satisfacción de la necesidad de autonomía de los sujetos al disponer de feedback inmediato y la interacción entre los participantes. El fomento de las relaciones sociales fue coincidente al estudio desarrollado por Consolvo, Everitt, Smith y Landay (2006) a través del uso de podómetros. La ausencia de mejora en la satisfacción de autonomía puede ser debida al nivel insuficiente de conocimiento de los métodos de entrenamiento de los participantes. Estas carencias no les permitieron desarrollar de forma independiente un programa de mejora desde el comienzo del mismo. El escaso uso del perfil creado en la red social o la pertenencia a diferentes centros pudieron provocar el descenso en la satisfacción de las relaciones sociales.

Seguidamente, se planteaba que el nivel de autodeterminación de los sujetos hacia la realización de actividad física sería mayor con el uso de la aplicación. Se han encontrando diferencias, aunque no significativas, en los resultados entre los dos grupos a la hora de tener una regulación más intrínseca hacia la realización de esta actividad a favor del grupo experimental. Estos mayores valores suponen a su vez un mayor aumento en la satisfacción de las necesidades de las necesidades psicológicas básicas (Zhang, Solmon, Kosma, Carson, y Gu, 2011). La evolución similar en algunas de las regulaciones puede haberse debido a la escasa duración en el tiempo de la intervención. Se ha de tener en cuenta que esta intervención ha servido como punto de partida para el desarrollo de un programa de fomento de actividad física extraescolar con las aplicaciones móviles como recurso fundamental de práctica.

Por último, se trató de comprobar el efecto del uso de la aplicación sobre la diversión. Existen diferencias en cuanto 
a la evolución del nivel de diversión en favor del grupo experimental, sobre todo teniendo en cuenta que el punto de partida era claramente inferior al grupo de control, no así en el aburrimiento. Se ha demostrado que mayores niveles de autodeterminación conllevan mayores niveles de diversión (García-Calvo, Sánchez-Miguel, Leo, Sánchez-Oliva, y Amado, 2012; Taylor, Ntoumanis, Standage, y Spray, 2010). El hecho de que los valores de aburrimiento sean significativos en ambos grupos puede ser consecuencia de la propia tendencia o actitud seguida de las clases de educación física. Este hecho pudo causar que los miembros del grupo experimental no llegaran a habituarse al uso de la aplicación, algo que repercutió en esta variable. Una mayor continuidad en el uso sistemático de esta herramienta podría dar como resultado mayores diferencias entre los grupos en este aspecto.

\section{Aplicaciones prácticas}

Nuestros resultados, a pesar de la escasez de la muestra y los escasos cambios producidos por la intervención en los resultados, nos aportan indicios que pueden definir futuras posibilidades de trabajo. Existen tendencias de que este tipo de tecnología permite obtener mejoras en cuanto a la satisfacción de las necesidades psicológicas básicas, y en el nivel de autodeterminación para la realización de actividad física aeróbica. De este modo, las aplicaciones de telefonía móvil de registro y control del entrenamiento pueden suponer un recurso didáctico más para los educadores de la etapa de Secundaria. De este modo, se pueden convertir en herramientas que apoyarían sus esfuerzos en la instauración en sus alumnos de hábitos saludables. Mediante este tipo de aplicación, la percepción del progreso en el desarrollo de actividades sería más directa, pudiendo repercutir en la satisfacción de competencia de los sujetos. Igualmente, la opción de compartir y publicar registros y datos dispone a los participantes para establecer y consolidar la comunicación con otros practicantes, pudiendo satisfacer la necesidad psicológica de relación social. Se dispone de un amplio margen de trabajo para futuras investigaciones en esta línea. Ciertos elementos de esta investigación, a pesar de no haber arrojado datos significativos, pueden ser valorados como líneas de actuación en proyectos presentados a instituciones públicas. En este sentido, cabe destacar el Programa Movil-Ízate, del grupo ADFEX con la ayuda de la Consejería de Salud y Política Social, del Gobierno de Extremadura (Orden de 6 de marzo de 2013). Se trata de un programa de intervención educativo desarrollado con alumnos de enseñanza secundaria utilizando las Tecnologías de la Información y Comunicaciones (TICs) para fomentar la práctica deportiva extraescolar de los jóvenes escolares. Concretamente, creó un concurso virtual con la colaboración de los centros de la Red Extremeña de Escuelas Promotoras de Salud, que permitiera llevar un seguimiento de la realización de práctica de extraescolar, a través de una aplicación móvil de control de entrenamiento.

Agradecimientos: Se manifiesta agradecimiento al profesorado colaborador en el estudio y a Endomondo Sport Tracker.

\section{Referencias}

1. Alvarez, M. S., Balaguer, I., Castillo, I., y Duda, J. L. (2009). Coach autonomy support and quality of sport engagement in young soccer players. The Spanish journal of psychology, 12, 138-48.

2. Balaguer, I., Atienza, F. L., Castillo, I., Moreno, Y., y Duda, J. L. (1997b). Factorial structure of measures of satisfaction/interest in sport and classroom in the case of Spanish adolescents. Abstracts of 4th. European Conference of Psychological Assessment (p. 76). Lisbon: Portugal.

3. Beltrán-Carrillo, V. J., Molina-Alventosa, J. P., y Valencia-Peris, A. (2011). Los videojuegos activos y la salud de los jóvenes: Revisión de la investigación. Revista Internacional de Medicina y Ciencias de la Actividad Fisica y del Deporte/International Journal of Medicine and Science of Physical Activity and Sport, 11(41), 203-219.

4. Biddle, S., Gorely, T., Marshall, S., Murdey, I., y Cameron, N. (2003). Physical activity and sedentary behaviours in youth: issues and controversies. The Journal of The Royal Society for the Promotion of Health, 124(1), 29-33.

5. Cecchini, J. A., González, C., y Contreras, O. (2004). Relaciones entre clima motivacional, la orientación de meta, la motivación intrínseca, la auto-confianza, la ansiedad y el estado de ánimo en jóvenes deportistas. Psicothema, 16(1), 104-109.

6. Cervelló, E. M., Escartí, A., y Guzmán, J. F. (2007). Youth sport dropout from the achievement goal theory. Psicothema, 19(1), 65-71.

7. Consolvo, S., Everitt, K., Smith, I., y Landay, J. A. (2006). Design requirements for technologies that encourage physical activity. In Proceedings of the SIGCHI conference on Human Factors in computing systems (pp. 457-466). ACM.

8. Deci, E. L., \& Ryan, R. M. (1995). Human autonomy. In Efficacy, agency, and self-esteem (pp. 31-49). Springer US.

9. Deci. E. L,. y Ryan, R. M. (2000). The "what" and "why" of goal pursuits: Human needs and the self-determination of behaviour. Psychological Inquiry, 11, 227-268.

10. Decreto 83/2007, de 24 de abril, por el que se establece el Currículo de Educación Secundaria Obligatoria para la Comunidad Autónoma de Extremadura. DOE: 7 de Mayo de 2007. (51). 7980-8152.

11. Decreto $115 / 2008$, de 7 de junio, por el que se establece el Currículo de Bachillerato en Extremadura. DOE: 18 de Junio de 2008. (117). 1635916644.

12. Duda, J. L., y Nicholls, J. G. (1992). Dimensions of achievement motivation in schoolwork and sport. Journal of educational psychology, 84(3), 290-299.

13. Cecchini, J. A., González-Mesa, C., Méndez, A., Fernández-Río, J., Contreras, O., y Romero, S. (2008). Metas sociales y de logro, persistencia-esfuerzo e intenciones de práctica deportiva en el alumnado de Educación Física. Psicothema, 20(2), 260-265.

14. Fanning, J., Mullen, S. , y McAuley, E. (2012). Increasing physical activity with mobile devices: a meta-analysis. Journal of medical Internet research, 14(6). 
15. García-Calvo, T., Sánchez-Miguel, P. A., Leo, F. M., Sánchez-Oliva, D., y Amado, D. (2012). Análisis del grado de diversión e intención de persistencia en jóvenes deportistas desde la perspectiva de la teoría de la autodeterminación. Revista de Psicología del Deporte, 21(1), 7-13.

16. Generelo, E., y Otros. (2004): Un maletín de pulsómetros para la educación física escolar. Grupo de investigación EFYPAF. Universidad de Zaragoza. VI Congreso Internacional de Educación Física y deporte escolar. FEADEF. Córdoba.

17. González-Cutre, D., Sicilia, Á., y Fernández, A. (2010). Hacia una mayor comprensión de la motivación en el ejercicio físico: medición de la regulación integrada en el contexto español. Psicothema, 22, (4) 841-847.

18. Jang, H., Reeve, J., y Deci, E. L. (2010). Engaging students in learning activities: It is not autonomy support or structure but autonomy support and structure. Journal of Educational Psychology, 102, 588-600.

19. Janz, K., y Mahoney, L. (1997). Maturation, gender, and video game playing are related to physical activity intensity in adolescents: The Muscatine Study. Pediatric Exercise Science, 9(4), 353-363.

20. King, A., Hekler, E., Grieco, L., Winter, S., Sheats, J., Buman, M., Banerjee, B., Robinson, T., y Cirimele, J. (2013). Harnessing different motivational frames via mobile phones to promote daily physical activity and reduce sedentary behavior in aging adults. PloS one, 8(4), e62613.

21. Kranz, M., Möller, A., Hammerla, N., Diewald, S., Plötz, T., Olivier, P., y Roalter, L. (2012). The mobile fitness coach: Towards individualized skill assessment using personalized mobile devices. Pervasive and Mobile Computing, 9(2), 203-215.

22. Martínez Baena, A. C., Chillón, P., Martín-Matillas, M., Pérez López, I., Castillo, R., Zapatera, B., Vicente-Rodríguez, G., Casajús, J. A., Álvarez-Granda, L., Romero, C., Cerezo, P. T. y Delgado Fernández, M. (2012) Motivos de abandono y no práctica de actividad físicodeportiva en adolescentes españoles: estudio Avena. Cuadernos de Psicología del Deporte, vol. 12, n. 1 (junio), 45-54.

23. Nunnally, J. C., y Bernstein, I. H. (1994). Psychometric theory (3a ed.). New York: McGraw-Hill.

24. Moreno, J. A., y González-Cutre, D. (2006). Predicción del disfrute en el ejercicio físico según la orientación disposicional y la motivación autodeterminada. Análisis y Modificación de Conducta, 32, 767-780.

25. Motl, R. W., McAuley, E., Birnbaum, A. S., y Lytle, L. A. (2006). Naturally occurring changes in time spent watching television are in versely related to frequency of physical activity during early adolescence. Journal of Adolescence, 29(1), 19-32.

26. Mulas, F., Pilloni, P., y Carta, S. (2012). Everywhere Race!: A Social Mobile Platform for Sport Engagement and Motivation. In SOTICS 2012, The Second International Conference on Social Eco-Informatics (pp. 63-69).

27. Murillo, B., García Bengoechea, E., Julián, J. A., y Generelo, E. (2014). Empowering adolescents to become physically active: three-year results of the "Sigue la Huella" intervention. Preventive Medicine, 66, 6-11.

28. Naylor, P. J., Macdonald, H. M., Zebedee, J. A., Reed, K. E., y McKay, H. A. (2006). Lessons learned from Action Schools! BC-an 'active school'model to promote physical activity in elementary schools. Journal of Science and Medicine in Sport, 9(5), 413-423.
29. Nunnally, J., y Bernstein, I. (1994). Psychometric theory (3rd ed.). New York: McGrawHill.

30. Orden de 6 de marzo de 2013 por la que se convocan subvenciones para la financiación de proyectos sociosanitarios en el campo de la promoción y educación para la salud, durante el año 2013. DOE: 25 de Marzo de 2013 (58). 6876-6895.

31. Roman, B., Serra-Majem, L., Pérez-Rodrigo, C., Drobnic, F., y Segura, R. (2009). Physical activity in children and youth in Spain: future actions for obesity prevention. Nutrition reviews, 67(1), 94-S98.

32. Ryan R. M., y Deci, E. L. (2000). Self-determination theory and the facilitation of intrinsic motivation, social development and well-being. American Psychologist, 55(1), 68-78.

33. Sánchez Miguel, P. A., Leo Marcos, F. M., Sánchez Oliva, D., Amado Alonso, D., \& García Calvo, T. (2012). La influencia del entorno próximo sobre la persistencia en la práctica de actividad físico-deportiva. Cuadernos de psicología del deporte, 12(1), 17-24.

34. Sánchez-Oliva, D., Amado, D., Chamorro, J. M., González-Ponce, I., y García-Calvo, T. (2013). Predicción de los Comportamientos Positivos en Educación Física: una perspectiva desde la Teoría de la Autodeterminación. En prensa.

35. Sánchez-Oliva, D., Leo, F. M., Sánchez-Miguel, P. A., Amado, D., y García-Calvo, T. (2013). Desarrollo de un modelo causal para explicar los comportamientos positivos en las clases de educación física. Acción Motriz, 10, 48-58.

36. Sánchez, J. M., y Núńez, J. L. (2007). Análisis preliminar de las propiedades psicométricas de la versión española de la Escala de Necesidades Psicológicas Básicas en el Ejercicio Físico. Revista Iberoamericana de Psicología del Ejercicio y el Deporte, 2(2), 83-92.

37. Sarrazin, P., Vallerand, R., Guillet, E., Pelletier, L., y Cury, F. (2002) Motivation and dropout in female handballers: A 21-month prospective study. European Journal of Social Psychology, 32(3), 395-418.

38. Taylor, I. M., Ntoumanis, N., Standage, M., y Spray, C. M. (2010) Motivational predictors of physical education students' effort, exercise intentions, and leisure-time physical activity: A multilevel linear growth analysis. Journal of Sport and Exercise Psychology, 32(1), 99-120.

39. Vallerand, R. J., y Rousseau, F. L. (2001). Intrinsic and extrinsic motivation in sport and exercise: A review using the hierarchical model of intrinsic and extrinsic motivation. Handbook of sport psychology, 2, 389-416.

40. Vickey, T. A., y Breslin, J. G. (2011). A Study on Twitter Usage for Fitness Self-Reporting via Mobile Apps. AAAI Technical Report SS-12-05.

41. Vlachopoulos, S. P., y Michailidou, S. (2006). Development and initial validation of a measure of autonomy, competence, and relatedness in exercise: The Basic Psychological Needs in Exercise Scale. Measurement in Physical Education and Exercise Science, 10(3), 179-201.

42. Wilson, P.M., Rodgers, W.M., Loitz, C.C., y Scime, G. (2006). «It's who I am ... really!». The importance of integrated regulation in exercise contexts. Journal of Applied Biobehavioral Research, 11, 79-104.

43. Zhang, T., Solmon, M. A., Kosma, M., Carson, R. L., y Gu, X. (2011). Need Support, Need Satisfaction, Intrinsic Motivation, and Physical Activity Participation among Middle School Students. Journal of teaching in physical education, 30(1), 51-68. 